\title{
Self-assessment questions: The acute porphyrias
}

\author{
David C Rees
}

1 Which of the following are potential precipitants of an attack of acute porphyria?
(a) Cimetidine
(b) Hormone replacement therapy
(c) Pregnancy
(d) Progesterone-only pill
(e) Menopause

2 Which of the following are characteristic symptoms of an acute attack of porphyria?
(a) Bipolar depression
(b) Motor neuropathy
(c) Sensory neuropathy
(d) Leg pain
(e) Hyponatraemia

3 Which of the following statements about haem are true?
a) Haem is essential for every cell in the body
b) Dietary haem intake from red meat reduces the rate of hepatic haem synthesis
c) Most haem is synthesised in the erythron
d) Haem deficiency explains most of the symptoms of acute porphyria
e) The rate of hepatic haem synthesis is increased in acute intermittent porphyria

4 Which of the following are useful in treating variegate porphyria?
a) Intravenous $5 \%$ dextrose
b) Gonadotrophin-releasing hormone agonists
c) Phenytoin
d) Liver transplantation
e) Opaque sun screen

Answers to these self-assessment questions can be found on page $s 92$. 